\title{
Suprimento vascular arterial do timo em aves (Gallus gallus domesticus) da linhagem Master Gris Cou Plumé
}

\author{
Eduardo Maurício Mendes de Lima ${ }^{1 *}$ \\ Martha de Oliveira Bravo ${ }^{1}$ \\ André Rodrigues da Cunha Barreto Vianna ${ }^{1}$ \\ Frederico Ozanam Carneiro e Silva ${ }^{2}$ \\ Renato Souto Severino ${ }^{2}$ \\ Sérgio Salazar Drummond ${ }^{2}$ \\ Marcelo Ismar Silva Santana ${ }^{3}$ \\ ${ }^{1}$ Faculdade de Agronomia e Medicina Veterinária, Universidade de Brasília \\ ICC, Ala Sul, Campus Universitário Darcy Ribeiro \\ Caixa Postal 4508, CEP 70910-970, Brasília - DF, Brasil \\ ${ }^{2}$ Faculdade de Medicina Veterinária \\ Universidade Federal de Uberlândia, Uberlândia - MG, Brasil \\ ${ }^{3}$ Pontifícia Universidade Católica, Poços de Caldas - MG, Brasil \\ *Autor para correspondência \\ limaemm@unb.br
}

Submetido em 08/10/2008

Aceito para publicação em 24/03/2009

\section{Resumo}

Para ampliar as informações sobre a morfologia comparativa, foram estudados, em aves (Gallus gallus domesticus) da linhagem Master Gris Cou Plumé, dados sobre o número de lobos tímicos, as origens, o número e a forma de distribuição das artérias que promoveram o suprimento sanguíneo deste órgão. Foram utilizadas 30 aves, 15 machos e 15 fêmeas, de aproximadamente seis semanas de idade, doadas, após morte natural, por criatórios do Distrito Federal. Os contingentes arteriais foram marcados com solução aquosa, a 50\%, de Neoprene Látex “450”, corada com pigmento específico. Posteriormente, as aves foram fixadas e conservadas em solução aquosa, a 10\%, de formaldeído, mediante aplicações intramusculares profundas, subcutâneas e intracavitárias e mantidas submersas na mesma solução. Foram encontrados de quatro a nove lobos tímicos cervicais e de um a dois lobos torácicos no antímero esquerdo; já no antímero direito, encontraram-se de três a sete lobos tímicos cervicais e de um a dois lobos torácicos. Verificou-se que os lobos tímicos foram supridos por ramos indiretos das artérias carótida comum, ingluvial, tireóidea caudal, tireóidea cranial, esofágica ascendente, comum do nervo vago e occipital, e ainda de ramos cutâneos. Foram supridos ainda por ramos diretos provindos das artérias ingluvial, tireóidea cranial e comum do nervo vago. Foi possível verificar que as aves desta linhagem apresentaram características próprias reforçadas ainda pelo fato das mesmas terem arranjos particulares para cada um dos indivíduos.

Unitermos: Gallus gallus domesticus, morfologia, timo, vascularização 


\section{Abstract}

Arterial vascular supply of the thymus in poultry (Gallus gallus domesticus) of the Master Gris Cou Plumé lineage. Seeking to expand information on the comparative morphology of poultry (Gallus gallus domesticus) of the Máster Gris Cou Plumé lineage, a study was conducted to provide data on the number of lobes of the thymus in addition to origin, number and distribution of the arteries that promoted the sanguineous supply of this organ. To this end 30 poultry ( 15 males and 15 females) were used. They were about six week old and were donated after natural death by poultry farms of the Federal District. The arterial supply was injected with a " 450 " Neoprene Latex stained solution. Subsequently, the poultry were fixed in a 10\% formoldehyde aqueous solution by means of deep intramuscular, subcutaneous and intracaviteous applications and were kept in the same solution. Four to nine cervical lobes were found, in addition to one or two thoracic lobes on the left side. On the right side, three to seven cervical lobes and one or two thoracic lobes were found. The lobes were supplied indirectly by branches coming from the common carotidal artery, the cranial and caudal thyroidal arteries, the ascending esophageal artery, the ingluvial artery, the common vagus nerve artery, the occipital artery and the cutaneous branches. They were also supplied by direct by branches from the ingluvial artery, cranial thyroidal artery and common vagus nerve artery. It was possible to verify that poultry of this breed not only possessed characteristics that were defining for the breed as such, but also demonstrated particular arrangements for each of the individuals.

Key words: Gallus gallus domesticus, morphology, thymus, vascularization

\section{Introdução}

A imunidade corporal está associada com atividades relacionadas aos órgãos linfomielóides e células, sendo o sistema linfático constituído de uma rede de defesa difusa. Nas aves, observa-se a presença de tecidos linfóides espalhados por todo o corpo e a ausência de linfonodos (Rose, 1979).

De acordo com a função e o desenvolvimento dos vários órgãos linfomielóides, pode-se classificálos em órgãos primários e centrais (Szenberg, 1976) ou secundários e periféricos (Glick, 1978). Os órgãos linfomielóides primários, produtores de precursores linfocíticos, incluem a medula óssea e o timo em diversas espécies animais, o saco vitelínico e a bolsa cloacal nas aves, estando principalmente centralizadas nestas e no timo as respostas imunológicas humorais em aves jovens (Kendall, 1980). De outra forma, são os órgãos secundários que promovem a maturação de células do sistema linfático, além de servirem como sítios das respostas imunes o baço, tonsilas cecais, glândula da terceira pálpebra, tonsilas palatinas e tecidos linfóides intestinais (Rose, 1979).

Os mecanismos que regulam o desenvolvimento deste órgão, assim como a linfocitopoiese, não estão definitivamente esclarecidos. Deve ser ressaltado que o timo é objeto de estudos profundos (Scala et al., 1984), mas que ainda tem sua morfologia pouco abordada e as circunstâncias que envolvem a sua regressão não esclarecida. Além disso, os componentes vasculares que promovem seu suprimento sanguíneo mostram-se como sendo um assunto pouco explorado, abordado de maneira genérica (Pereira, 1998; Gonçalez et al., 2000; Santana et al., 2000; 2001; Silva et al., 2001; Carvalho et al., 2004).

Com o propósito de aprimorar as informações a respeito da morfologia comparativa do timo, objetivase neste estudo abordar, em aves (Gallus gallus domesticus) da linhagem Master Gris Cou Plumé, por sua rusticidade e grande produtividade, os dados pertinentes à morfologia desse órgão e, ainda, as origens, o número e a forma de distribuição das artérias que promoveram o seu suprimento arterial.

\section{Material e Métodos}

Foram utilizados 30 exemplares de aves (Gallus gallus domesticus) da linhagem Master Gris Cou Plumé, 15 machos e 15 fêmeas, de aproximadamente seis semanas de idade, provindas de núcleos criatórios do Distrito Federal, após morte natural.

Os animais foram encaminhados ao Laboratório de Anatomia Veterinária da Faculdade de Agronomia 
e Medicina Veterinária da Universidade de Brasília, onde tiveram os seus contingentes arteriais marcados. Para isso, o sistema vascular arterial das mesmas foi preenchido com solução aquosa, a 50\%, de Neoprene Látex “450” (Du Pont do Brasil S/A-Indústria Química), corada com pigmento específico (Globo S/A Tintas e Pigmentos), a partir da canulação da artéria isquiática esquerda, em sentido cranial, com cânula de polietileno, de calibre compatível ao diâmetro do vaso.

Para a fixação e conservação das peças, foi utilizada solução aquosa, a 10\%, de formol (LABSYNTH Produtos para Laboratório Ltda), mediante aplicações intramusculares profundas, subcutâneas e intracavitárias e, em seguida, as aves foram mantidas submersas, na mesma solução, em recipientes adequados. Após 48 horas, procederam-se as dissecações das artérias responsáveis pelo suprimento sanguíneo do timo e, simultaneamente, avaliou-se o arranjo morfológico desse órgão.

Para a descrição dos resultados, considerou-se o timo de acordo com sua disposição torácica e cervical. $\mathrm{Na}$ dissecação dos ramos arteriais destinados ao timo, foram utilizados instrumentos cirúrgicos adequados, auxiliados pelo campo visual de uma lupa monocular.

Os modelos de suprimento sanguíneo do referido órgão, então encontrados em todos os espécimes estudados, foram transferidos esquematicamente para fichas individuais, registrando-se a origem, o número e a ordenação dos ramos arteriais. Foram documentados ainda os arranjos mais peculiares das artérias que supriram o timo, a partir de registros fotográficos. Promoveu-se ainda documentação relativa à morfologia do timo destes animais, em especial a disposição e número dos seus lobos.

\section{Resultados}

Diante dos achados morfológicos relativos ao timo das aves ora estudadas, foram encontrados de quatro a nove lobos cervicais e de um a dois lobos torácicos no antímero esquerdo. Já no antímero direito, foram encontrados de três a sete lobos cervicais e de um a dois lobos torácicos. A cadeia de lobos tímicos destes animais esteve disposta de maneira linear, paralela e lateral ao pescoço, em todos os casos e em ambos antímeros, como mostram as Figuras 1 e 2.

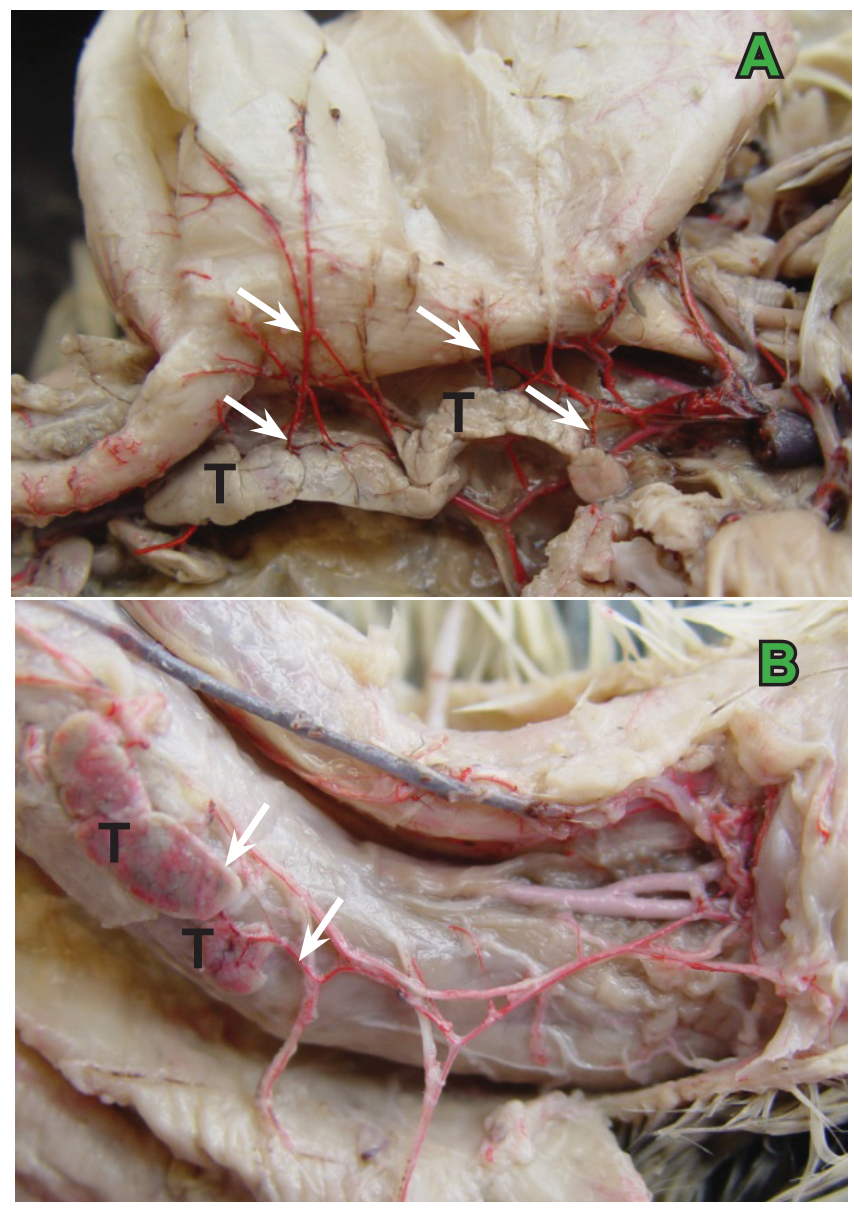

FIGURA 1: Fotografias representativas (A e B) do suprimento vascular arterial dos lobos tímicos $(\mathrm{T})$ dos antímeros esquerdo de aves da linhagem Máster Gris Cou Plumé, ilustrando os ramos das artérias ingluvial esquerda (setas pretas) e ramos cutâneos (setas brancas).

No que diz respeito ao suprimento arterial, o aspecto dos ramos destinados ao timo pode ser observado na Figura 1.

Neste estudo, foi observado que a artéria carótida comum contribuiu com um ramo direto, em três casos, no antímero esquerdo; dois ramos diretos, em um caso, e um ramo indireto, em dois casos, no mesmo antímero.

A artéria ingluvial cedeu um ramo direto, em quatro casos, no antímero esquerdo e, no antímero direito, cedeu um ramo direito, em dois casos, além de um ramo indireto, em um caso. 


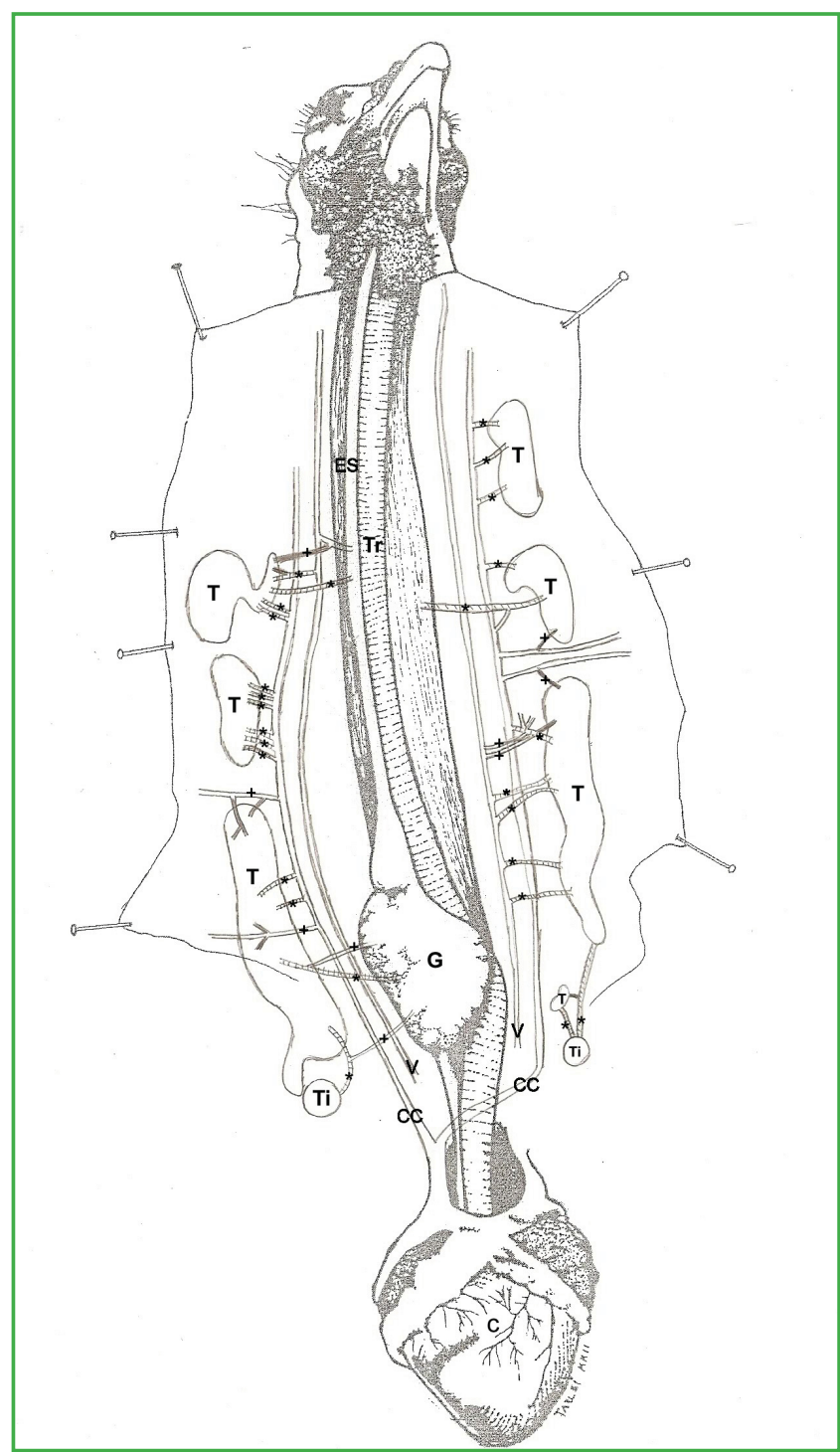

FIGURA 2: Desenho esquemático representativo do suprimento vascular arterial dos lobos tímicos (T) dos antímeros esquerdo de aves da linhagem Máster Gris Cou Plumé, ilustrando os ramos diretos $(+)$ e indiretos $\left(^{*}\right)$ cedidos pelas artérias carótida comum (CC) e comum do nervo vago $(\mathrm{V})$.

A artéria tireóidea caudal contribuiu com um ramo direto, em seis casos, dois ramos diretos, em um caso, e um ramo indireto, em cinco casos, no antímero esquerdo. Já a artéria tireóidea cranial contribuiu com um ramo direto, em 12 casos, dois ramos diretos, em um caso, e três ramos diretos, em um caso, no antímero esquerdo, assim como com um ramo indireto, em oito casos, e dois ramos indiretos, em um caso, para o mesmo antímero. No antímero direito, esta artéria cedeu um ramo direto, em 18 casos, e um ramo indireto em seis casos.
Em relação à artéria esofágica ascendente, esta emitiu sete ramos diretos, em um caso, no antímero esquerdo e, no antímero direito, contribuiu fornecendo dois ramos diretos, em dois casos, três ramos diretos, em sete casos, quatro ramos diretos, em três casos, cinco ramos diretos, em seis casos, seis ramos diretos, em três casos, sete ramos diretos, em um caso, nove ramos diretos, em três casos e 11 ramos diretos, em um caso.

A artéria comum do nervo vago contribuiu, no antímero esquerdo, com ramos diretos para o timo, sendo que liberou sete ramos diretos, em três casos, oito ramos diretos, em quatro casos, nove ramos diretos, em três casos, dez ramos diretos, em cinco casos, 11 ramos diretos, em três casos, 13 ramos diretos, em três casos, 14 ramos diretos, em quatro casos e 18 ramos diretos, em um caso. De outra forma, emitiu, para o mesmo antímero, um ramo indireto, em nove casos, dois ramos indiretos, em seis casos, três ramos indiretos, em três casos, quatro ramos indiretos, em um caso e cinco ramos indiretos, em um caso. Por sua vez, no antímero direito, foi visto que a artéria comum do nervo vago contribuiu cedendo seis ramos diretos, em três casos, oito ramos, em três casos, nove ramos, em seis casos, dez ramos, em três casos, 11 ramos, em um caso, 12 ramos, em quatro casos, 13 ramos, em um caso, 14 ramos, em três casos, 15 ramos, em três casos e 16 ramos, em um caso, além de um ramo indireto, em cinco casos, dois ramos indiretos, em quatro casos, três ramos indiretos, em dois casos e quatro ramos indiretos, em um caso.

Dos ramos cutâneos diretos, do antímero esquerdo, originou-se um ramo para o timo, em um caso, dois ramos, em quatro casos, três ramos, em cinco casos, quatro ramos, em nove casos, cinco ramos, em dois casos, seis ramos, em um caso, sete ramos, em um caso, oito ramos, em um caso e nove ramos, em um caso. No antímero direito, dos ramos cutâneos diretos originouse um ramo, em três casos, dois ramos, em oito casos, três ramos, em oito casos, quatro ramos, em dois casos, cinco ramos, em quatro casos, seis ramos, em dois casos e sete ramos, em um caso.

A artéria occipital cedeu para o timo, no antímero esquerdo, um ramo indireto, em dois casos e dois ramos indiretos, em um caso. Já no antímero direito, a mesma artéria cedeu dois ramos diretos, em um caso. 


\section{Discussão}

Apesar da existência de pesquisas relativas à morfologia macroscópica do timo das aves, e de muitas vezes este órgão ser objeto de estudos intensos, algumas informações pertinentes ao tema foram tratadas de maneira superficial pelos autores, principalmente com relação ao suprimento sanguíneo para os lobos tímicos (Pereira, 1998; Gonçalez et al., 2000; Santana et al., 2000; 2001; Silva et al., 2001; Carvalho et al., 2004).

Estando os lobos tímicos localizados lateralmente no pescoço, estes apresentaram íntima relação com os nervos vagos e as veias jugulares, de ambos antímeros, dispostos assim ao longo dos seus trajetos na região cervical desta espécie, concordando com os relatos prestados por Szenberg (1976), Kendall (1980), Gonçalez et al. (1997), Pereira (1998) e Santana et al. (2000; 2001).

De outra forma, como verificado no presente estudo, foi observada a ocorrência de lobos torácicos do timo, como descrito por Santana et al. (2001), nas aves investigadas neste estudo .

Conforme descrito por Scala et al. (1984), Gonçalez et al. (1997), Pereira (1998) e Santana et al. (2000; 2001), os lobos tímicos de ambos os antímeros, em todos os casos ora estudados, apresentaram tamanhos e formatos variáveis, bem como o número de lobos.

De acordo com os relatos de Santana et al. (2000; 2001), a glândula tireóide das aves investigadas neste estudo apresentou-se envolvida em um dos lobos tímicos, em ambos os antímeros, em todos os casos.

No que diz respeito ao suprimento arterial para os lobos tímicos da linhagem Master Gris Cou Plumé, a maioria dos ramos encontrados foram diretos $\mathrm{e}$ indiretos da artéria comum do nervo vago, o que pode ser explicado pela íntima relação daquele nervo com os lobos tímicos (Santana et al., 2008). Porém, também foram encontrados ramos diretos e indiretos das artérias carótida comum, ingluvial, tireóidea cranial, tireóidea caudal, esofágica ascendente, comum do nervo vago, ramos cutâneos e artéria occipital, concordando assim com os relatos de Gonçalez et al. (1997), Pereira (1998) e Santana et al. (2000; 2001; 2008).
Os ramos diretos das artérias que suprem o timo apresentaram pontos variáveis de penetração. Dessa forma, não ficou evidenciada a presença de um hilo tímico, como descrito por Pereira (1998) e Santana et al. $(2000 ; 2001)$.

De acordo com os ramos arteriais que supriram o timo das aves em questão, para cada um dos antímeros, verificou-se que entre estes não existiu cruzamento de ramos arteriais - entre antímeros opostos - em relação aos lobos supridos, conforme descrito por Gonçalez et al. (1997), Pereira (1998) e Santana et al. (2000; 2001).

\section{Referências}

Carvalho, V. B.; Santana, M. I. S.; Silvino, M. J.; Bombonato, P. P.; Benedicto, H. G.; Silva, F. O. C. 2004. Thymus morphology on domestic pigeons (Columbia livia gmelin). International Journal of Morphology, 22 (1): 35-37.

Glick, B. 1978. The immune response in the chicken: Lymphoid development of the bursa of Fabricus and thymus and an immune response role for the gland of Harder. Poultry Science, 57: 14411444.

Gonçalez, P. O.; Silva, F. O. C.; Drummond, S. S.; Severino, R. S.; Bombonato, P. P.; Machado, G. V. 2000. Suprimento arterial do timo de Gallus gallus da linhagem Peterson. Arquivos de Ciências Veterinárias e Zoologia da Unipar, 3 (1): 47-54.

Gonçalez, P. O.; Silva, F. O. C.; Severino, R. S.; Santos, A. L. Q.; Drummond, S. S.; Bombonato, P. P.; Santana, M. I. S. 1997. Suprimento arterial do timo em aves (Gallus gallus domesticus - linhagem Peterson). Anais do XXV Congresso Brasileiro de Medicina Veterinária, Gramado, Brasil, p.106.

Kendall, M. D. 1980. Avian thymus gland. Developmental and Comparative Immunology, 4: 191-209.

Pereira, C. C. H. 1998. Irrigação arterial do timo em aves (Gallus gallus domesticus) da linhagem Hubbard. Dissertação de Mestrado, Universidade de São Paulo, Brasil, 72pp.

Rose, E. 1979. The immune system in birds. Journal of the Royal Society of Medicine, 72: 701-705.

Santana, M. I.; Bombonato, P. P.; Araújo, L. V.; Castello, A. P. F.; Escanhoela, T. B. 2008. Irrigação do timo em aves da linhagem Paraíso Pedrês (Gallus gallus domesticus). Arquivo Brasileiro de Medicina Veterinária e Zootecnia, 60 (2): 307-314.

Santana, M. I. S.; Carneiro e Silva, F. O.; Drummond, S. S.; Severino, R. S.; Bombonato, P. P. 2001. Vascularização arterial do timo em aves (Gallus gallus domesticus - Linnaeus, 1758) da linhagem NPK. Veterinária Notícias, 7 (1): 13-19.

Santana, M. I. S.; Carneiro e Silva, F. O.; Drummond, S. S.; Severino, R. S.; Bombonato, P. P.; Santos, A. L. Q. 2000. Vascularização arterial do timo em aves (Gallus gallus domesticus) da linhagem Avian Farms. Bioscience Journal, 16 (2): 3-15.

Scala, G.; Langella, M.; Budetta, G. 1984. Sulla vascularizzazione del timo nell anatra. Bollettino della Società Italiana di Biología Sperimentale, 60 (4): 701-706. 
Silva, F. O. C.; Severino, R. S.; Drummond, S. S.; Perissoto, D. O.; Campos, D. B.; Campos, A. B. 2001. Artérias do timo de aves (Gallus gallus) da linhagem Label Rouge. Bioscience Journal, 17 (2): 115-123.

Szenberg, A. 1976. Ontogenesis of the immune system in birds. In: Marchabris, J. J. (Ed.). Comparative immunology. Blackwell Scientific Publications, Oxford, UK, p.20. 